\title{
In vivo monitoring of cystic fibrosis-like lung disease in mice by volumetric computed
} tomography

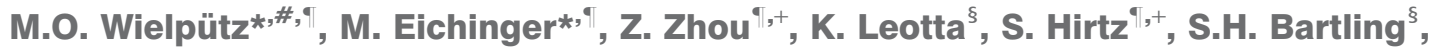

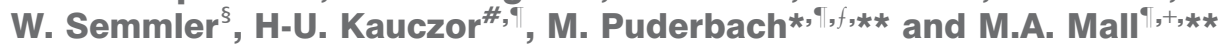

ABSTRACT: The onset and spontaneous development of cystic fibrosis (CF) lung disease remain poorly understood. In the present study, we used volumetric computed tomography (VCT) as a new method for longitudinal in vivo monitoring of early lesions and disease progression in CF-like lung disease in $\beta$-epithelial $\mathrm{Na}^{+}$channel (ENaC)-transgenic (TG) mice.

Using a VCT scanner prototype (80 kV, $50 \mathrm{~mA} \cdot \mathrm{s}$, scan time $19 \mathrm{~s}$ and spatial resolution $200 \mu \mathrm{m})$, BENaC-TG mice and wild-type (WT) littermates were examined longitudinally at 10 time-points from neonatal to adult ages, and VCT images were assessed by qualitative and quantitative morphological parameters.

We demonstrate that VCT detected early-onset airway mucus obstruction, diffuse infiltrates, atelectasis and air trapping as characteristic abnormalities in $\beta E N a C-T G$ mice. Furthermore, we show that early tracheal mucus obstruction predicted mortality in $\beta E N a C-T G$ mice and that the density of lung parenchyma was significantly reduced at all time-points in $\beta E N a C-T G$ compared with WT mice (median \pm SEM $-558 \pm 8 \mathrm{HU}$ in WT versus $-686 \pm 16 \mathrm{HU}$ in $\beta E N a C-T G$ at 6 weeks of age; $\mathbf{p}<0.005)$.

Our study demonstrates that VCT is a sensitive, noninvasive technique for early detection and longitudinal monitoring of morphological abnormalities of CF-like lung disease in mice, and may thus provide a useful tool for pre-clinical in vivo evaluation of novel treatment strategies for CF.

KEYWORDS: Computed tomography, cystic fibrosis, epithelial $\mathrm{Na}^{+}$channel, lung disease, lung imaging, transgenic mouse model

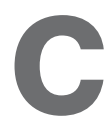
ystic fibrosis (CF) lung disease is caused by mutations in the cystic fibrosis transmembrane conductance regulator (CFTR) gene and is one of the most common lethal hereditary diseases in Caucasians [1, 2]. The hallmarks of CF lung disease are highly viscous airway secretions causing airway mucus obstruction, chronic inflammation, refractory bacterial infection, bronchiectasis and emphysema leading to progressive lung destruction and respiratory failure $[3,4]$.

In epithelial cells lining the airway surfaces, CFTR acts as a cyclic AMP-dependent $\mathrm{Cl}^{-}$channel and regulator of the epithelial $\mathrm{Na}^{+}$channel $(\mathrm{ENaC})$ [58], thus regulating epithelial transport of salt and water and the height of the thin film of liquid that covers the airways (airway surface liquid (ASL)). In CF airways, CFTR dysfunction results in deficient $\mathrm{Cl}^{-}$secretion and increased $\mathrm{ENaC}-$ mediated $\mathrm{Na}^{+}$ absorption, producing ASL depletion and impaired mucociliary clearance [9-11]. The critical role of ASL depletion in the in vivo pathogenesis was confirmed in the $\beta \mathrm{ENaC}$-transgenic (TG) mouse, which mimics increased airway $\mathrm{Na}^{+}$absorption and develops a CF-like lung disease characterised by high pulmonary mortality due to airway mucus obstruction and chronic inflammation, poor bacterial clearance, and emphysema with distal airspace enlargement and increased lung capacity in adult survivors [12-14].

Previous studies using the $\beta E N a C-T G$ mouse to monitor the in vivo development of morphological changes in CF-like lung disease, and the use of this model for pre-clinical evaluation of novel therapeutic strategies, relied on post mortem histopathology $[12,13,15]$. This approach is inherently limited by single time-point observations, and no data are available from $\beta \mathrm{ENaC}-\mathrm{TG}$ mice that die spontaneously of lung disease during the
AFFILIATIONS

*Dept of Radiology,

${ }^{\S}$ Medical Physics in Radiology, German Cancer Research Center (DKFZ),

\#Dept of Diagnostic and Interventional Radiology, +Division of Paediatric Pulmonology and Allergy, and Cystic Fibrosis Center, Dept of Paediatrics III, ${ }^{f}$ Dept of Diagnostic and Interventional Radiology with Nuclear Medicine, Thoraxklinik, University of Heidelberg,

"Translational Lung Research Center, Heidelberg, Germany.

**These authors contributed equally to the study.

CORRESPONDENCE

M.A. Mall

Division of Paediatric Pulmonology and Allergy, and Cystic Fibrosis Center

Dept of Paediatrics III

University of Heidelberg and

Translational Lung Research Center Heidelberg

Im Neuenheimer Feld 430

69120 Heidelberg

Germany

E-mail: Marcus.Mall@med.uni-

heidelberg.de

Received:

Sept 232010

Accepted after revision:

March 202011

First published online:

April 082011 
observational period. Furthermore, for longitudinal studies, large numbers of mice are needed to study interindividual lung morphology at different time-points [13]. Other investigators have used micro-computed tomography $(\mu \mathrm{CT})$ as an alternative approach to study lung morphology in murine models of interstitial lung disease and emphysema in vivo and post mortem, at very high resolution $[16,17]$. However, $\mu \mathrm{CT}$ has not been used for longitudinal in vivo studies due to the stressful and potentially lethal procedure, which requires long scanning times ( $\leqslant 30 \mathrm{~min}$ ), high radiation doses and invasive anaesthesia $[18,19]$. Volumetric computed tomography (VCT) provides the possibility for intraindividual longitudinal imaging studies to evaluate the natural course of lung disease in individual mice in vivo, at a spatial resolution high enough to study lung morphology in small laboratory animals [20,21]. Instead of the multidetector rows used in clinical scanners, VCT uses a rotating flat-panel detector, achieving isotropic voxels of $\sim 200 \mu \mathrm{m}$ with the setup used for the present study. In contrast to $\mu \mathrm{CT}, \mathrm{VCT}$ is less stressful for experimental animals because of shorter acquisition times (scan time $\sim 19 \mathrm{~s}$ ) and, therefore, lower cumulative exposure to X-rays and anaesthetics. Additionally, a large field of view allows examination of multiple mice within the same scan [20-22].

The aim of this study was to evaluate VCT to monitor the onset and spontaneous progression of lung disease in $\beta \mathrm{ENaC}-\mathrm{TG}$ mice in vivo, and to describe radiological features of this histomorphologically well-characterised mouse model of CF lung disease. To achieve this goal, we performed longitudinal VCT imaging studies in $\beta E N a C$-TG mice and wild-type (WT) littermate controls from neonatal to adult ages, and compared airway and parenchymal morphology by qualitative evaluation of airway mucus obstruction, infiltrates, atelectasis and air trapping, and by quantitative measurements of lung density. Findings from VCT studies were validated by lung histology, morphometric analyses of airway mucus obstruction and distal airspace enlargement, and pulmonary function tests at selected time-points.

\section{MATERIALS AND METHODS}

\section{Experimental animals}

All animal studies were approved by the Regierungspräsidium (Karlsruhe, Germany). The generation of $\beta E N a C-T G$ mice (line 6608) has been described previously [12]. The colony was originally established and maintained on a mixed genetic background $(\mathrm{C} 3 \mathrm{H} / \mathrm{HeN} \times \mathrm{C} 57 \mathrm{BL} / 6 \mathrm{~N})$. Experimental animals were generated by breeding of hemizygous $\beta \mathrm{ENaC}$-TG mice with C3B6 F1 WT mice (Charles River Laboratories, Sulzfeld, Germany) to prevent genetic drift in the colony, and $\beta \mathrm{ENaC}$ TG mice were identified by PCR of genomic DNA as described previously [12, 13]. WT littermates served as controls in all experiments to control for possible effects of genetic background and environment on the phenotype. Mice were housed in a specific pathogen-free animal facility, and had free access to food and water.

\section{Volumetric computed tomography}

A prototype VCT scanner (Siemens Medical Solutions AG, Forchheim, Germany) consisting of a flat-panel detector and a modified X-ray tube, both mounted on a clinical multislice CT gantry, was employed as previously described [22, 23]. The flat-panel detector (PaxScan 4030CB; Varian Medical Systems, Palo Alto, CA, USA) poses 2,048 $\times 1,536$ detector pixels on an active area of $40 \times 30 \mathrm{~cm}^{2}$, each with a dimension of $194^{2} \mu \mathrm{m}^{2}$. The source-to-detector distance was $93.0 \mathrm{~cm}$ and the source-toisocentre distance was $57.3 \mathrm{~cm}$, resulting in a magnification of 1.6. The detector was read out in a $2 \times 2$ binning mode, meaning that four neighbouring pixels were averaged. Taking the geometry of the VCT system setup into consideration, the instrument's total field of view was $25 \times 25 \times 18 \mathrm{~cm}^{3}$ at an isotropic resolution of $200 \mu \mathrm{m}[22,23]$. The frame rate was 30 frames $\cdot \mathrm{s}^{-1}$. Scan parameters were $80 \mathrm{kV}$ and $50 \mathrm{~mA} \cdot \mathrm{s}$ at a scan time of $19 \mathrm{~s}$. The effective dose of a scan that employs the same scan parameters measured in air kerma was $\sim 22.8 \mathrm{mGy}$ [23]. Mice were placed randomly side by side in a prone position for scanning in groups of three littermates. VCT was performed in freely breathing mice under inhalative sedation with 1-2\% sevoflurane (Sevorane ${ }^{\circledR}$; Abbott, Wiesbaden, Germany) nebulised in pure $\mathrm{O}_{2}$ (Linde, Pullach, Germany) on days $3,5,7,10,14,17,21,24,28$ and 42 after birth, or immediately after death. As up to three mice were examined at the same time, the field of view for the reconstruction in growing mice was adapted to the individual chest diameter. Image reconstruction in the transverse plane from $360^{\circ}$ projection data was performed with a cone-beam algorithm by filtered back-projection, with a sharp kernel typically used for lung imaging, as described previously [23].

\section{VCT image evaluation}

Evaluation of VCT images was performed by an investigator (M.O. Wielpütz) blinded for the genotype of the mice in a lung window (width 1,240 HU; center -400 HU) (Chili Pacs® Software version 2.6; Chili GmbH, Heidelberg, Germany), using a commercially available workstation with two flat-panel screens. The software allows simultaneous image evaluation in three planes by real-time multiplanar reconstruction. This approach enables secure identification of the lesions of interest as well as partial volume effects, which is beneficial, for example, for the analysis of airway obstruction. To facilitate comparative viewing in the figures, VCT images of mice of different ages were magnified to the same size, which explains the lower final resolution (relative blurring) of images shown from young mice. Scale bars were added in each image to indicate differences in lung size. Initial VCT images of mice of all ages were scanned for distinct lesions, which were used to develop a standardised database to evaluate VCT images with respect to qualitative and quantitative parameters as follows.

\section{Qualitative assessment}

Airway obstruction was assessed separately in the trachea, main stem bronchi and intrapulmonary bronchi. Partial or complete opacification of the tracheal lumen between larynx and bifurcation, main stem bronchi or intrapulmonary bronchi was rated as a positive finding. In addition to airway obstruction, each quadrant of the lung parenchyma was independently assessed for the presence of the following abnormalities: 1) diffuse infiltrates, defined as diffusely distributed spotted opacities of the lung not covering a lobe or segment; 2) atelectasis, defined as homogeneous opacity with volume loss that covered a lobe or segment; and 3) air trapping, defined as a focal, sharply delineated area of decreased density compared with the encompassing parenchyma. Furthermore, the overall 
visual appearance of the lung texture as a whole was classified as homogeneous, focally inhomogeneous (showing at least one of the lesions defined above) or globally inhomogeneous.

\section{Quantitative assessment}

The density of the lung parenchyma was measured in Hounsfield units (HU) by placing four circular regions of interest (ROIs) with a diameter of $1 \mathrm{~mm}$ in both the upper and lower lung regions. When placing the ROIs, a representative lung area was selected randomly after excluding large airways, large vessels, infiltrates, atelectasis and air trapping from the measurement. Based on these four measurements, the mean density was calculated for each mouse.

\section{Morphology}

Anaesthetised mice were killed by exsanguination. Lungs and tracheae were removed through a median sternotomy, fixed in $4 \%$ buffered formalin and embedded in paraffin. Sections were cut at $5 \mu \mathrm{m}$ and stained with haematoxylin and eosin (H\&E) or Alcian blue periodic acid-Schiff (AB-PAS), as previously described [13].

\section{Airway morphometry}

Morphometric analyses of airway mucus obstruction were performed in noninflated, immersion-fixed tracheae and left lungs. Tracheae were sectioned longitudinally and lungs were sectioned transversally at the level of the proximal intrapulmonary main axial airway near the hilus [13]. For quantitative stereological assessment of airway mucus obstruction, we used the Cell`F image analysis software (Olympus $\mathrm{GmbH}$, Hamburg, Germany) to determine mucus volume density, as previously described [13, 24]. In brief, images of airway sections were acquired with an Olympus IX-71 microscope (Olympus GmbH), the length of the airway boundary, as defined by the epithelial basement membrane, was measured by the interactive image measurement tool and the AB-PAS-positive surface area within this boundary was measured by phase analysis according to the automatic threshold settings of the software. The volume density of airway mucus, representing the volume of airway mucus content per millimetre of surface area of the basement membrane $\left(\mathrm{nL} \cdot \mathrm{mm}^{-2}\right)$, was determined from the surface area of AB-PAS-positive mucus and the basement membrane length, as previously described [13, 24].

\section{Mean linear intercepts}

Lungs from 3-day-old mice were immersion-fixed in $4 \%$ buffered formalin, and right lungs from 2- and 6-week-old mice were inflated with $4 \%$ buffered formalin to $25 \mathrm{~cm}$ of fixative pressure. Subsequently, lungs were processed for histology, sectioned at $5 \mu \mathrm{m}$ and stained with H\&E. Histological images were captured digitally with an Olympus IX-71 microscope, using Cell^ ${ }^{\wedge} \mathrm{image}$ analysis software with a line counting tool at a 16-fold magnification, beginning at randomly selected spots. Mean linear intercepts were determined by dividing the sum of the lengths of all lines in all frames by the number of intercepts between alveolar septi and counting lines [25]. For each animal, a minimum of 200 intercepts were sampled in 10 fields in different lobes, as previously described [13].

\section{Pulmonary function studies}

To evaluate lung mechanics, 6-week-old adult mice were anaesthetised with an intraperitoneal injection of ketamine and xylazine (120 and $16 \mathrm{mg} \cdot \mathrm{kg}^{-1}$, respectively), paralysed by intraperitoneal injection of $0.8 \mathrm{mg} \cdot \mathrm{kg}^{-1}$ pancuronium bromide (Inresa Arzneimittel GmbH, Freiburg, Germany), tracheotomised, and mechanically ventilated at a rate of 150 breaths $\cdot \mathrm{min}^{-1}$, a tidal volume of $10 \mathrm{~mL} \cdot \mathrm{kg}^{-1}$ and a positive end-expiratory pressure of $3 \mathrm{cmH}_{2} \mathrm{O}$ with a computer-controlled small animal ventilator (FlexiVent system; SCIREQ Inc., Montreal, QC, Canada). Pressure-volume curves were obtained using the PVs-P (stepwise pressure-regulated) setting and total lung capacity (TLC) was determined using the custom-designed FlexiVent software (SCIREQ Inc.), as previously described [26].

\section{Statistical analysis}

The data were recorded in a dedicated database (Microsoft Access; Microsoft Corp., Redmond, WA, USA). Statistical analysis was performed with SigmaPlot ${ }^{\circledR}$ (Systat Software $\mathrm{GmbH}$, Erkrath, Germany). Dichotomous data from qualitative parameters were tested pairwise using Fisher's exact test and correction for multiple testing was performed by Bonferroni's method. Qualitative data are presented as percentages of mice per group presenting the lesion of interest. For quantitative data, normality testing was performed to assess whether parametric or nonparametric tests should be employed. Multiple comparisons of quantitative data obtained by VCT or morphometry at different time-points in mice of the same genotype (i.e. WT or $\beta E N a C-T G$ ) were performed using oneway ANOVA or Kruskal-Wallis ANOVA on ranks with post hoc tests as appropriate. Statistical comparisons between mice of different genotypes (i.e. WT versus $\beta \mathrm{ENaC}-\mathrm{TG}$ ) were performed by unpaired t-test or Mann-Whitney U-test and multiple comparisons were compensated for by using Bonferroni's method. Quantitative data are presented as mean or median $\pm \mathrm{SEM}$, or median (interquartile range). A p-value of $<0.05$ or $<0.05 / m$ (number of tests) for Bonferroni's method was considered statistically significant. Additionally, receiver operating characteristic (ROC) analysis was performed for density data (in HU) in order to determine a threshold that discriminates between WT and $\beta E N a C-T G$ mice. The positive likelihood ratio (LR+) was calculated. Cut-off values with a $\mathrm{LR}+$ of $>10$ are generally considered reliable.

\section{RESULTS}

\section{Development of airway mucus obstruction in $\beta E N a C-T G$ mice}

To study the longitudinal development of airway obstruction by VCT, we evaluated images of neonatal to adult $\beta \mathrm{ENaC}-\mathrm{TG}$ mice and WT littermates for partial or complete opacification of conducting airways, including the trachea (fig. 1), main stem bronchi (fig. 2) and intrapulmonary bronchi. In $\beta \mathrm{ENaC}$ TG mice, opacification of the trachea was a characteristic feature of early lung disease and the frequency of this lesion decreased with age (fig. 1d, e and g). In contrast, tracheal obstruction was not observed by VCT in WT mice at any timepoint (fig. 1a, b and g). Comparative histopathological evaluation of tracheae in 3-day-old neonatal mice demonstrated that opacities detected by VCT reflected intraluminal mucus obstruction (fig. 1c and f), as previously described [13]. Overall, tracheal obstruction was detected by VCT in five $(25 \%)$ out of $20 \beta \mathrm{ENaC}$-TG mice on day 3 , all of which died spontaneously before the end of the study (fig. $1 \mathrm{~g}$ and $\mathrm{h}$ ). 

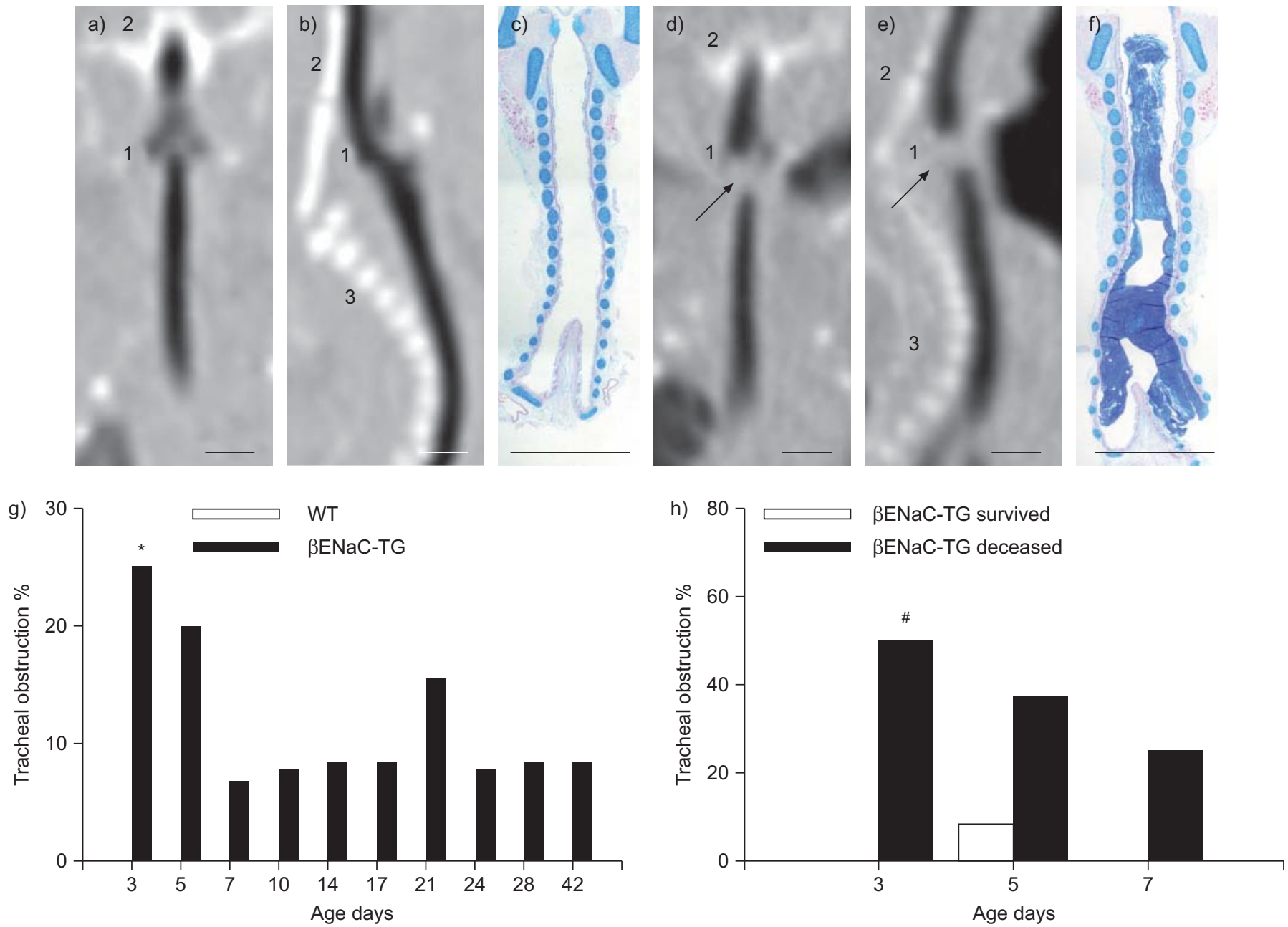

FIGURE 1. Early tracheal mucus obstruction in $\beta$-epithelial $\mathrm{Na}^{+}$channel (ENaC)-transgenic (TG) mice. Multiplanar reconstructions (MPRs) in a) coronal and b) sagittal planes of the neck of a 5-day-old wild-type (WT) mouse showing the larynx with its lateral recessus (1), head (2) and spine (3). The MPRs of a representative $\beta$ ENaC-TG mouse show obstruction of the sublaryngeal trachea (arrow) in the d) coronal and e) sagittal views. The mouse died spontaneously on day 6 . Notably, this is an example of how three-dimensional reconstruction can help to discriminate between the artificial blurring of the larynx in the WT mouse by partial volume effect and obstruction in the $\beta E N a C-T G$ mouse. Histology (Alcian blue periodic acid-Schiff staining) showing mucus obstruction of the trachea in $\mathrm{f}$ ) a representative $\beta E N a C-T G$ mouse killed at the age of 3 days but not in c) a WT mouse. Scale bars $=1 \mathrm{~mm}$. g) Longitudinal monitoring of the frequency of narrowing or complete obstruction of the trachea in $\beta E N a C$-TG mice by volumetric computed tomography. Note that tracheal obstruction was not observed in WT mice. h) Tracheal obstruction in deceased and long-term surviving $\beta E N a C-T G$ mice. Data are shown as percentage of mice with a lesion present at each time-point. $n=12-20$ mice per group. *: $p<0.05$ versus age-matched WT mice (Fisher's exact test); \#: $p<0.05$ versus age-matched $\beta E N a C-T G$ survivors (Fisher's exact test).

Subsequently, narrowing or complete obstruction of the main stem bronchi was observed by VCT in a substantial number of $\beta \mathrm{ENaC}-\mathrm{TG}$ mice and, to more variable extents, in WT littermates (fig. 2a-c and supplementary figure online). However, main stem bronchi of $\beta \mathrm{ENaC}-\mathrm{TG}$ mice were significantly more often affected than those of WT mice on day $7(p<0.05)$ and showed a trend towards a higher frequency of bronchial obstruction thereafter (fig. 2c). This pattern detected by VCT was consistent with the pattern of intraluminal mucus obstruction of main stem bronchi detected by lung histology in $\beta \mathrm{ENaC}-\mathrm{TG}$ and WT mice. In agreement with previous studies [13], airway morphology and morphometry at selected time-points of the VCT study demonstrated severe and persistent mucus obstruction in $\beta \mathrm{ENaC}-\mathrm{TG}$ mice, and a transient increase of goblet cell numbers and mucus accumulation in juvenile WT mice that peaked at the age of 2 weeks and waned thereafter (fig. 2d-h). Notably, the identification of obstruction of small intrapulmonary bronchi by VCT was limited by the maximum spatial resolution (i.e. $\sim 200 \mu \mathrm{m}$ ). Taken together, these data demonstrate that VCT is a sensitive technique for in vivo monitoring of mucus obstruction in conducting airways including trachea and main stem bronchi in mice.

\section{$\beta E N a C$-TG mice exhibit abnormalities of the lung parenchyma}

Next, we performed a longitudinal evaluation of focal and generalised abnormalities of the lung parenchyma in WT and $\beta E N a C-T G$ mice. Figure 3 shows representative VCT images of WT and $\beta E N a C-T G$ mice at four different time-points during lung development and provides an overview of characteristic abnormalities in $\beta \mathrm{ENaC}-\mathrm{TG}$ mice. First, we focused on focal 

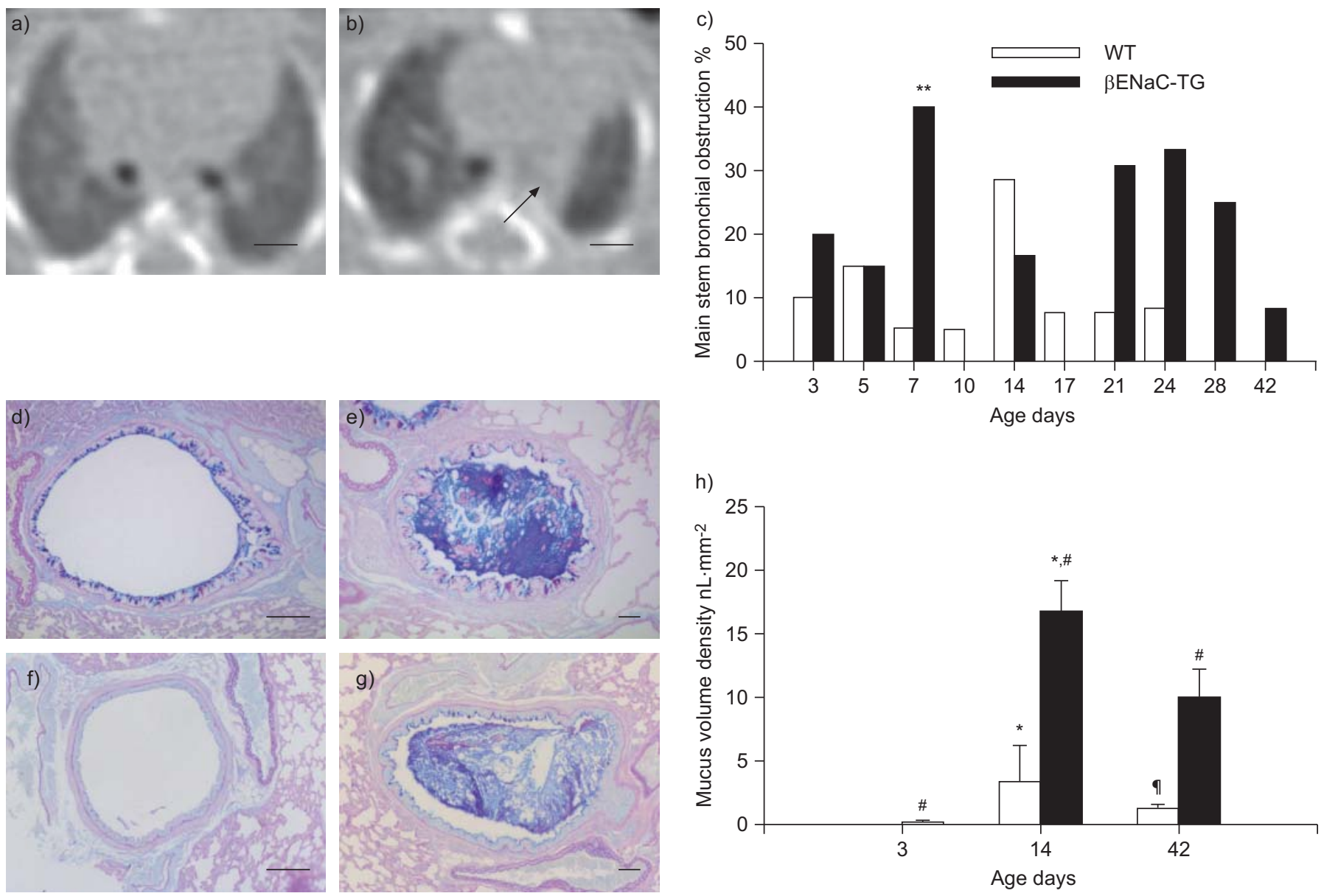

FIGURE 2. Mucus obstruction in main stem bronchi from wild-type (WT) and $\beta$-epithelial $\mathrm{Na}^{+}$channel (ENaC)-transgenic (TG) mice. a) Volumetric computed tomography (VCT) slice of a WT mouse on day 7. b) Obstruction of the left main stem bronchus (arrow) in a BENaC-TG mouse on day 7. Images are aligned at the level before the right main stem bronchus enters the lung. Scale bars $=1 \mathrm{~mm}$. c) Summary of longitudinal assessment of mucus obstruction in main stem bronchi in WT and $\beta E N a C-T G$ mice by VCT. Data are presented as the percentage of mice with lesion present. $n=11-20$ mice per group. ${ }^{*}: \mathrm{p}<0.01$ versus age-matched WT (Fisher's exact test). Representative airway histology (Alcian blue periodic acid-Schiff staining) from d, f) WT and e, g) $\beta$ ENaC-TG mice killed at the age of d, e) 2 and f, g) 6 weeks. Lungs were sectioned at the level of the proximal main axial airway near the hilus, showing transient goblet cell metaplasia and intraluminal mucus in d) 2-week-old but not f) 6-week-old WT mice, and persistent mucus obstruction in e, g) $\beta E N a C-T G$ mice. Scale bars $=100 \mu \mathrm{m}$. h) Mucus content in the proximal airways. Data are presented as median \pm SEM. $\mathrm{n}=5-9$ mice per group. *: $p<0.05$ versus mice of same genotype on day 3 (Kruskal-Wallis ANOVA with post hoc Dunn's method); $:$ p $<0.017$ versus age-matched WT (Bonferroni-corrected Mann-Whitney U-test); ${ }^{\top}: \mathrm{p}<0.05$ versus mice of same genotype day 14 (Kruskal-Wallis ANOVA with post hoc Dunn's method).

structural alterations of the lung parenchyma, including infiltrates, atelectasis and air trapping. In WT mice, infiltrates and atelectasis occurred only rarely and without any predilection for a specific age. Lucencies, a sign of air trapping, were not observed in WT mice and the global lung texture was homogeneous at all time-points (figs 3-5). In contrast, diffuse infiltrates were more frequently observed in $\beta E N a C-T G$ mice compared with WT littermates during the first week of life $(\mathrm{p}<0.05$ to $\mathrm{p}<0.001)$, but were rarely detected thereafter (fig. 4b, c and e). Signs of atelectasis were more commonly found in 1-3-week-old $\beta E N a C$-TG mice compared with WT controls ( $p<0.05$ on day 21) (fig. $4 \mathrm{c}, \mathrm{d}$ and $\mathrm{f}$ ). As shown in figure 5, air trapping occurred in parallel with atelectasis in $\beta \mathrm{ENaC}-\mathrm{TG}$ mice, where it was most frequent at the age of $7-$ 24 days (fig. 5a). Moreover, $\beta E N a C-T G$ mice presented with a globally inhomogeneous and hypodense lung structure from day 3 onwards (figs 3 and 5b). By considering the presence of inhomogeneous parenchyma as a single parameter, $\beta \mathrm{ENaC}-\mathrm{TG}$ mice could reliably be identified by the reader $(\mathrm{p}<0.005)$ (fig. 5b).

\section{Development of emphysema in $\beta E N a C-T G$ mice}

In addition to qualitative assessment of the lung texture by $\mathrm{VCT}$, we quantified the lung density of WT and $\beta E N a C-T G$ mice by measuring the CT density in Hounsfield units. In WT mice, these measurements revealed an increase in median density during the first days of life, with a peak at day 10 compared with day 3 after birth $(\mathrm{p}<0.05)$ (fig. 6a). After this peak, the density continuously decreased until the age of 42 days, when tissue density was significantly reduced compared with juvenile $(5-10$-day-old) WT mice $(\mathrm{p}<0.05)$. In $\beta E N a C-T G$ mice, density was significantly lower than in agematched WT mice at all time-points $(p<0.005)$. Furthermore, tissue density failed to increase in $\beta \mathrm{ENaC}$-TG mice during the first week of life, but decreased continuously from day 3 to day $42(p<0.05)$. As a consequence, the difference in lung 
WT
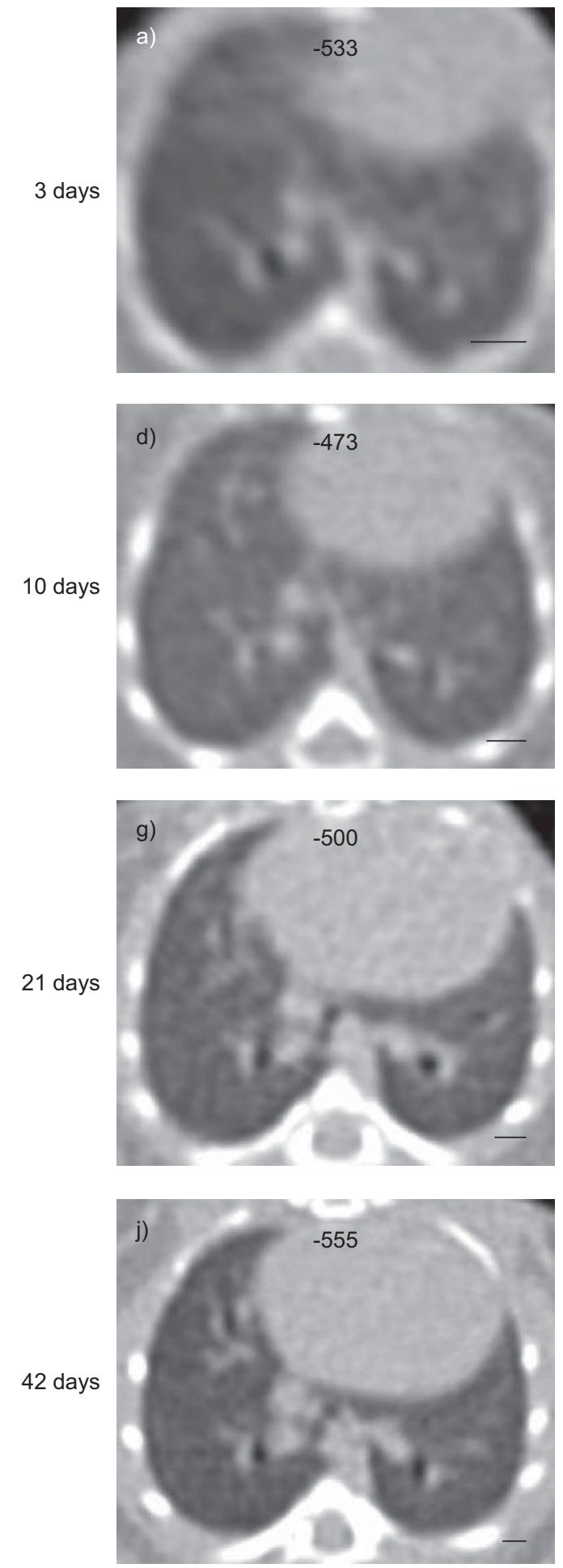

$\beta E N a C-T G$ survivor
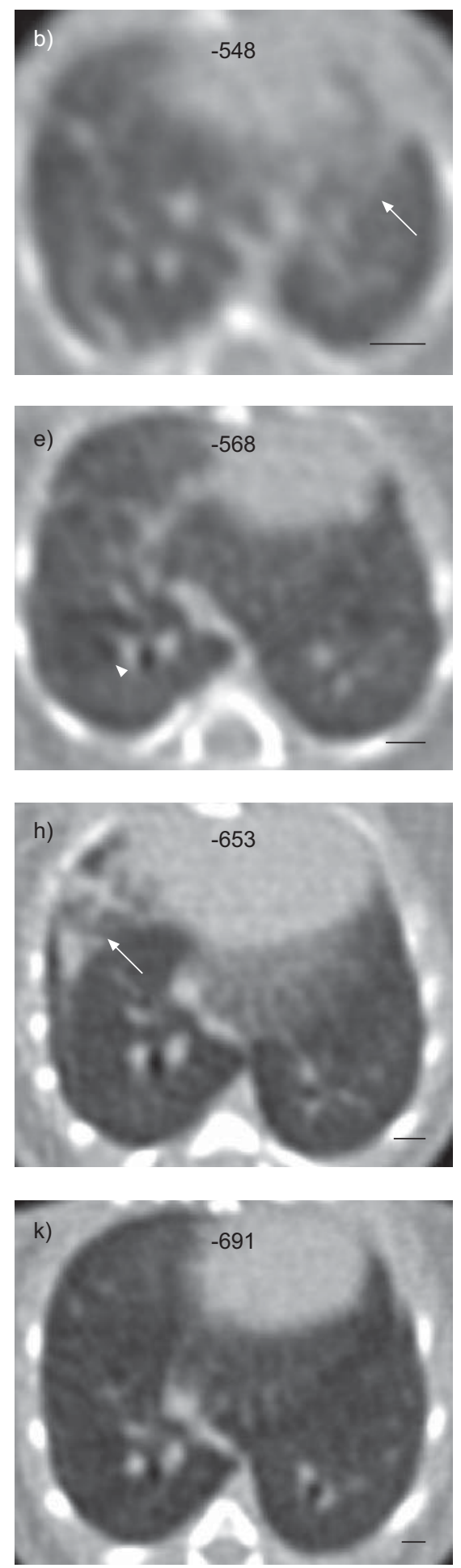

$\beta$ ENaC-TG deceased
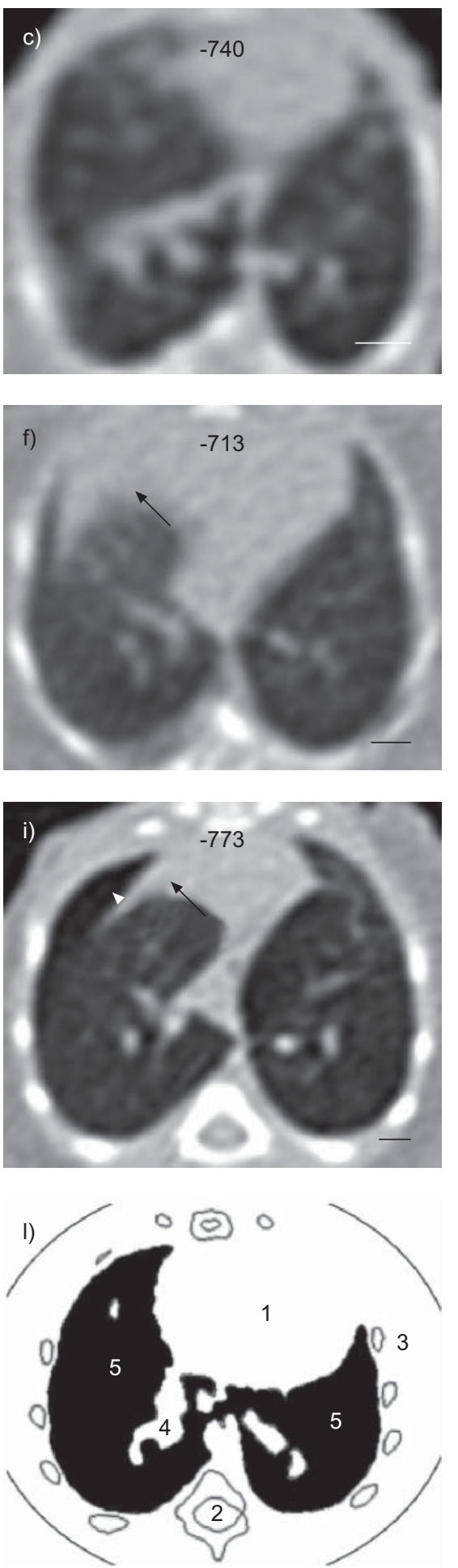

FIGURE 3. Representative longitudinal volumetric computed tomography (VCT) images of the chest of wild-type (WT) and $\beta$-epithelial $\mathrm{Na}^{+}$channel (ENaC)-transgenic (TG) mice. VCT images of the lung were taken sequentially starting at the age of 3 days in a, d, g, j) a WT mouse, b, e, h, k) a surviving $\beta E$ EaC-TG littermate and c, f, i) a $\beta E N a C-T G$ mouse that died spontaneously on day 21. Note the development of an overall inhomogeneous lung parenchyma in $\beta E N a C-T G$ mice compared with the homogeneous texture in the WT mouse, and a difference in emphysema severity in the two $\beta E N a C-T G$ mice. These images also give examples of other characteristic pathologies, such as diffuse infiltrates (white arrows), atelectasis (black arrows) and air trapping (white arrowheads). Corresponding mean lung densities (in Hounsfield units) for each mouse are given at the top of each image. Images were aligned at the level of the branching of the right inferior lobe bronchus. Scale bars=1 mm. I) Landmark structures of the mouse chest: heart (1), spine (2), chest wall with costae (3), bronchovascular branches (4) and lung parenchyma (5). 

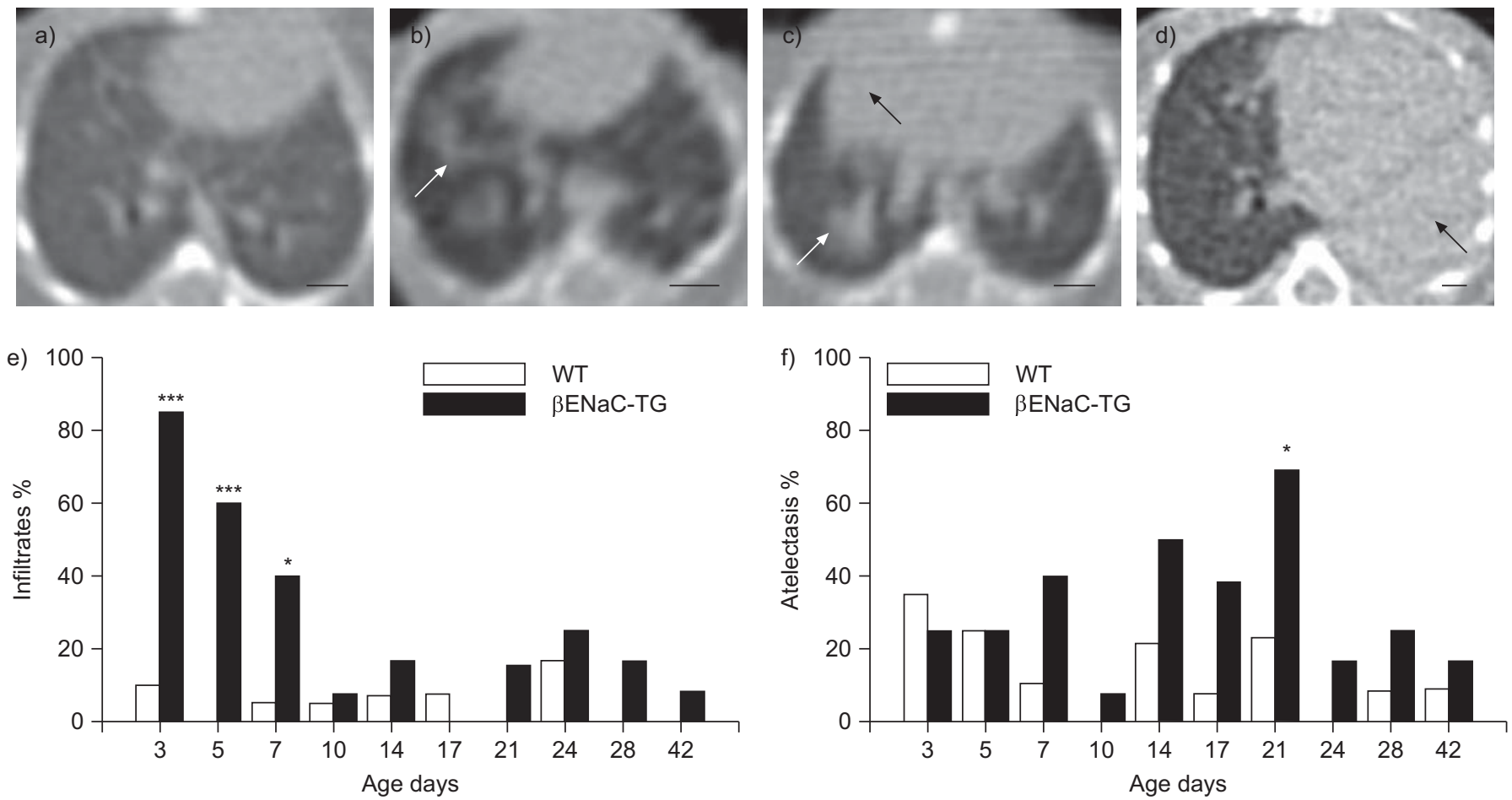

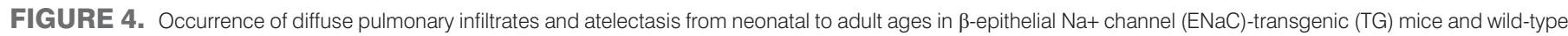

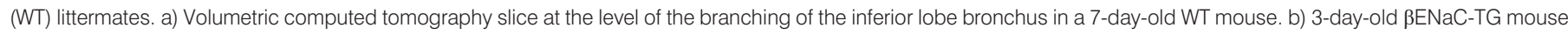

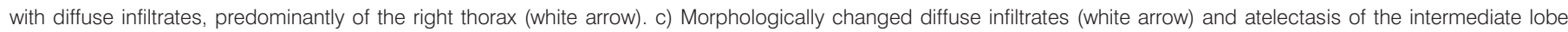

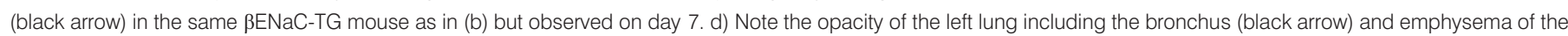

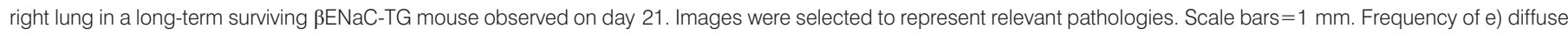

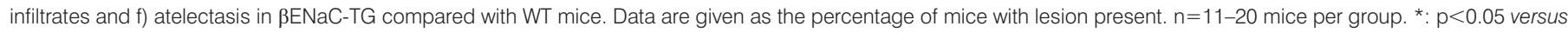
age-matched WT (Fisher's exact test); ${ }^{* * *}$ : $p<0.001$ versus age-matched WT (Fisher's exact test).

density between $\beta E N a C-T G$ and WT mice increased from the first to the last scan. On day 3 , the median \pm SEM difference in density between WT and $\beta E N a C-T G$ mice was $60 \pm 20 \mathrm{HU}$, and this gap increased significantly to $129 \pm 16 \mathrm{HU}$ on day 42 $(\mathrm{p}<0.05)$. ROC analysis revealed that as early as day $3, \mathrm{WT}$ and $\beta E N a C-T G$ mice can be discriminated by a cut-off value of $-552 \mathrm{HU}$ with a sensitivity of $79 \%$, a specificity of $95 \%$ and a LR+ of 15.8. On day 42 , sensitivity and specificity were calculated as 92 and $91 \%$, respectively, and the LR+ as 10.1 for a cut-off of -605 HU. Taken together, these results demonstrate substantial changes in parenchymal density during normal lung development in WT mice, and identify reduced tissue density characteristic of emphysema as an early and persistent abnormality in lungs from $\beta E N a C-T G$ mice. To validate these findings from VCT studies using independent measurements, we determined mean linear intercepts as a parameter for alveolar diameter at selected time-points (at 3, 14 and 42 days of age) (fig. 6b-d). These measurements demonstrated that reduced tissue density, as determined in Hounsfield units by VCT (fig. 6a), was associated with significant enlargement of distal airspaces at all time-points in $\beta E N a C-T G$ mice compared with WT littermates $(\mathrm{p}<0.017)$ (fig. $6 \mathrm{~b}-\mathrm{d}$ ). Furthermore, pulmonary function testing demonstrated a significant upwards shift of the pressure-volume curves $(\mathrm{p}<0.01)$ and a significant increase in TLC $(p<0.05)$, which are characteristic of emphysema [26], in adult $\beta E N a C-T G$ compared with WT mice (fig. 6e and f). Collectively, these data demonstrate that $\beta \mathrm{ENaC}-\mathrm{TG}$ mice develop early-onset emphysema and that VCT is a sensitive imaging modality to monitor emphysema development in vivo.

\section{DISCUSSION}

Lung disease in CF patients is characterised by various, often coexisting lesions, including airway mucus plugging, atelectasis, infiltration and emphysema [2-4]. However, little is known about the initial lesions and spontaneous development of disease in vivo, and currently, no therapies are available that target CF lung disease at its root cause. The $\beta E N a C-T G$ mouse mimics basic CF airway defects (i.e. increased airway $\mathrm{Na}^{+}$ absorption and ASL depletion) and phenocopies CF lung disease, providing an opportunity for studies of the in vivo pathogenesis and pre-clinical evaluation of novel therapeutic strategies [12, 13, 15, 27]. Previous studies with this mouse model of CF lung disease have provided a detailed histomorphological characterisation of airway mucus obstruction and emphysema $[12,13]$, but the onset and spontaneous progression of these morphological changes have not been studied in vivo.

Therefore, in this study, we used VCT to analyse the development and spontaneous progression of CF-like lung disease in $\beta E N a C$-TG mice, and validated data obtained from VCT studies by histomorphological studies and pulmonary function testing at selected time-points. In agreement with previous histology studies [13] and histomorphological data from this study (figs 1 and 2), VCT imaging detected a high 

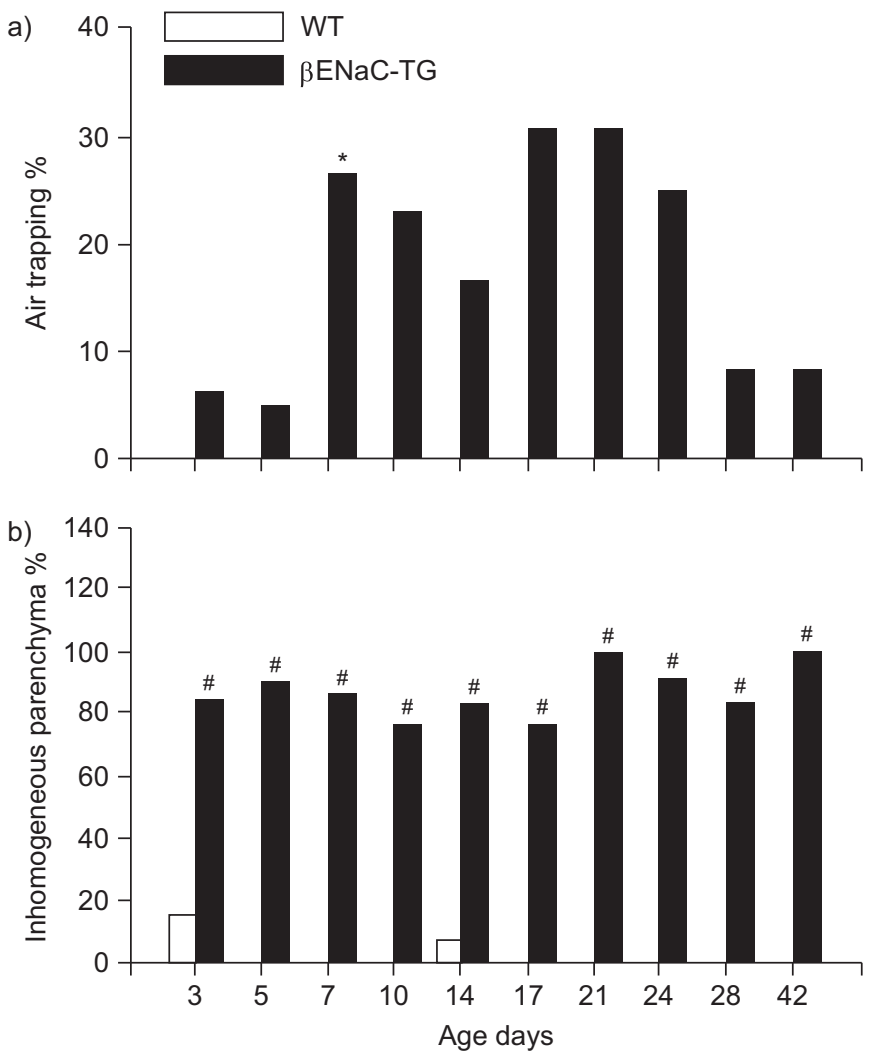

FIGURE 5. Summary of longitudinal assessment for a) air trapping and b) diffuse parenchymal inhomogeneity in $\beta$-epithelial $\mathrm{Na}^{+}$channel $(\mathrm{ENaC})$-transgenic (TG) and wild-type (WT) mice. a) Percentage of $\beta E N a C-T G$ mice showing air trapping. Note that air trapping was not observed in WT mice. b) Inhomogeneous lung parenchyma was found in most $\beta E N a C-T G$ mice, but was rarely detected in WT littermates. Data are presented as the percentage of mice with lesion present. $\mathrm{n}=11-21$ mice per group. ${ }^{*}: \mathrm{p}<0.05$ versus age-matched WT (Fisher's exact test); \#: $p<0.005$ versus age-matched WT (Bonferroni-corrected Fisher's exact test).

incidence of tracheal opacification, reflecting early mucus plugging of the trachea in the neonatal period, and progression of airway mucus obstruction into the lower airways of $\beta \mathrm{ENaC}$ TG mice in the first weeks of life. Notably, early post-natal obstruction of the trachea was observed in $\beta \mathrm{ENaC}-\mathrm{TG}$ but not in WT mice and was a strong predictor of subsequent death of affected mice. The inter- and intraindividual variability of airway obstruction observed between different time-points suggests that airway narrowing detected by VCT reflected variable obstruction due to intraluminal accumulation of mucus in the airways of $\beta \mathrm{ENaC}-\mathrm{TG}$ mice.

In addition to airway mucus obstruction, VCT depicted several structural abnormalities of the lung parenchyma in $\beta E N a C-T G$ mice that were not appreciated in previous histomorphological studies. In general, focal structural abnormalities of the lung, subdivided into diffuse infiltrates, atelectasis and air trapping were significantly more frequent in BENaC-TG mice compared with WT littermates (figs 4 and 5). Regarding the timing of the occurrence of these abnormalities during development, we speculate that the early appearance of diffuse infiltrates in $\beta \mathrm{ENaC}-\mathrm{TG}$ mice may be a consequence of central mucus plugging in the trachea, leading to a more global impairment of ventilation [13]. Conversely, we speculate that the later onset of atelectasis and air trapping (figs 4 and 5) reflects a more regional pattern of mucus obstruction of intrapulmonary bronchi in $\beta E N a C-T G$ mice beyond the first week of life (fig. 2).

Furthermore, we detected a significantly reduced tissue density (fig. 6a) and global parenchymal inhomogeneity (fig. 5b) in $\beta E N a C-T G$ compared with WT mice at all stages. In WT mice, density increased significantly until day 10 , probably reflecting the process of alveolar septation, leading to an increase in tissue density during early post-natal development [28]. Subsequently, parenchymal tissue density gradually decreased, as expected during the growth of the thorax and concomitant distension of distal airspaces. In $\beta E N a C-T G$ mice, parenchymal density was significantly reduced at all time-points studied, and the difference in tissue density between $\beta \mathrm{ENaC}-\mathrm{TG}$ and WT mice increased with age during the observational period. Interestingly, the difference in lung density between WT and $\beta \mathrm{ENaC}$ TG mice was similar to the difference recently observed between chronic obstructive pulmonary disease (COPD) patients with emphysema and controls [29], indicating that $\beta E N a C-T G$ mice develop emphysema to a level that is pathophysiologically relevant in humans. Reduced parenchymal tissue density in $\beta E N a C-T G$ mice was associated with early-onset distal airspace enlargement, as determined from measurements of mean linear intercepts in neonatal to adult mice (fig. 6d). Furthermore, pulmonary function tests detected abnormalities characteristic of emphysema, such as an upwards shift of the pressurevolume curves and increased TLC [26], in $\beta \mathrm{ENaC}-\mathrm{TG}$ mice compared with WT littermates (fig. 6e and f). Taken together, these data obtained from VCT imaging, alveolar morphology and lung function testing demonstrated that early-onset and progressive emphysema is a characteristic abnormality in $\beta$ ENaC-TG mice.

Regarding the mechanism of emphysema formation, we speculate that several factors may contribute to the early development of this abnormality in $\beta \mathrm{ENaC}-\mathrm{TG}$ mice, including: 1) impaired post-natal alveolarisation; 2) persistent air trapping due to mucus obstruction, leading to irreversible mechanical over-distension of distal airspaces; and 3) a protease/antiprotease imbalance resulting from chronic inflammation associated with recruitment of macrophages and neutrophils and elevated levels of pro-inflammatory cytokines [13]. In this context, ongoing studies indicate that airway surface dehydration may cause activation of macrophages by particulates or irritants that are not properly cleared from mucostatic airways, causing elevated expression of macrophage elastase (matrix metalloprotease 12), which may be implicated in emphysema formation in $\beta E N a C-T G$ mice [30-32]. Notably, besides small airway mucus obstruction, early-onset emphysema has been reported as an early and invariable feature in the lungs of CF infants who died in the first year of life [3], highlighting the clinical importance of this phenotype in CF patients. Our studies demonstrate that VCT imaging provides a powerful tool for in vivo monitoring of emphysema in mice. We expect that this noninvasive end-point will help to further elucidate the mechanisms underlying emphysema formation in CF and other chronic lung diseases in future pre-clinical studies, including genetic and pharmacological approaches. 
a)
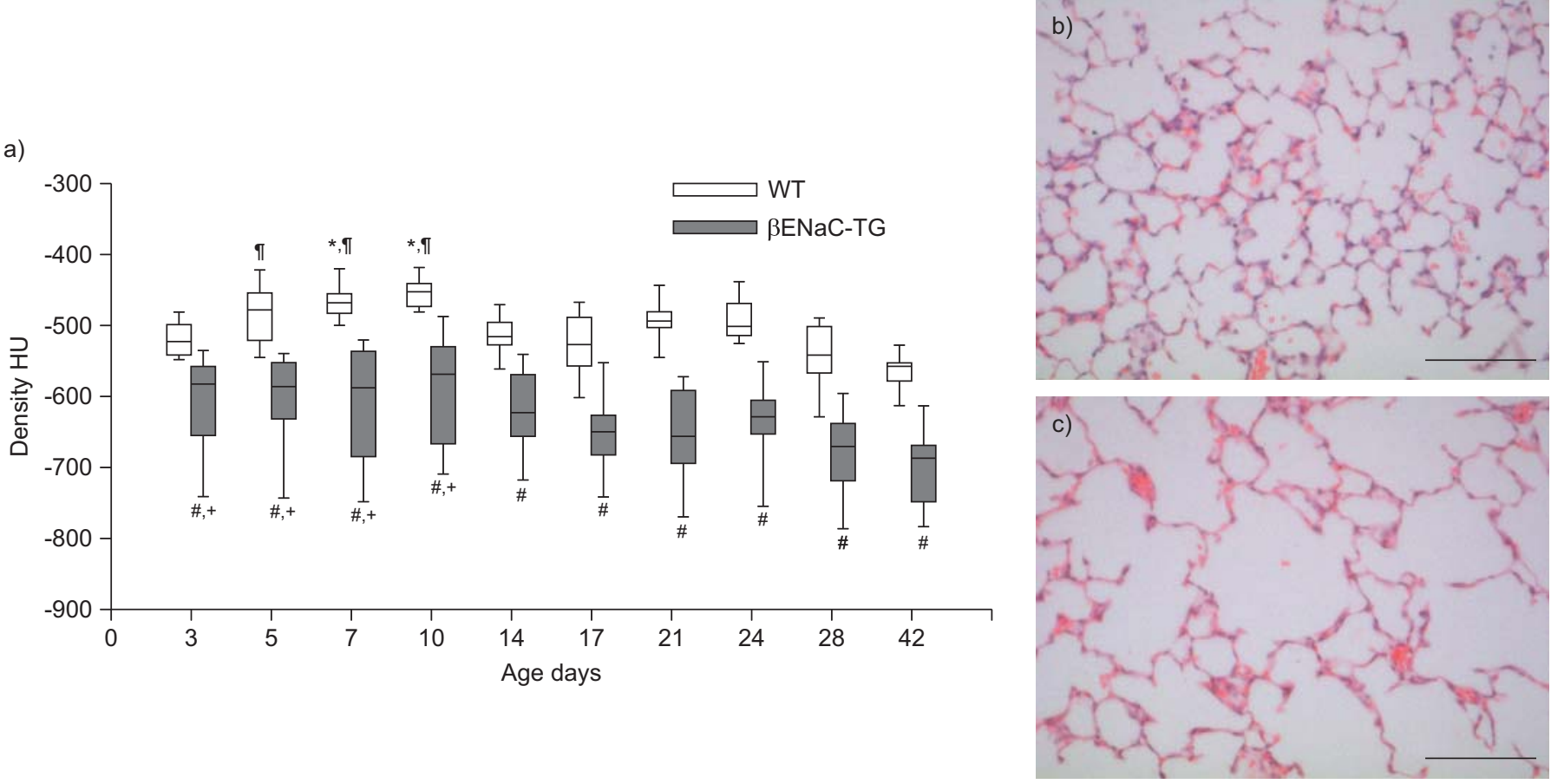

d)

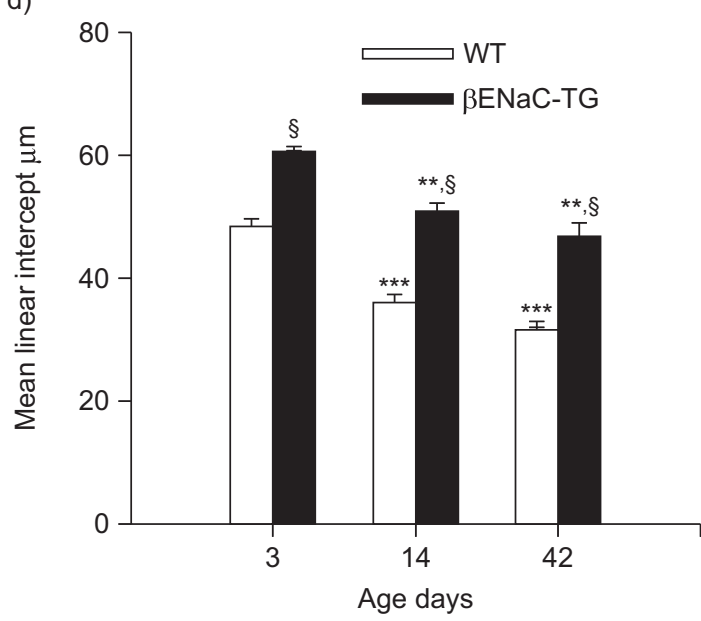

e)

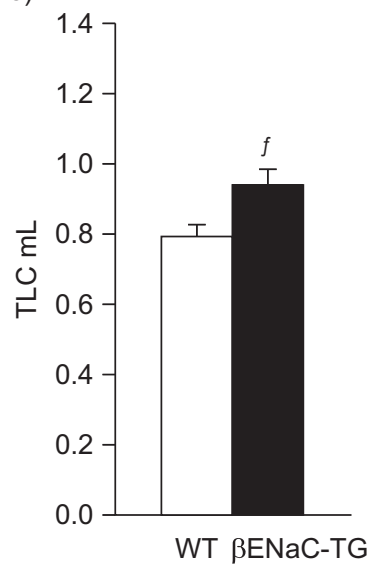

f)

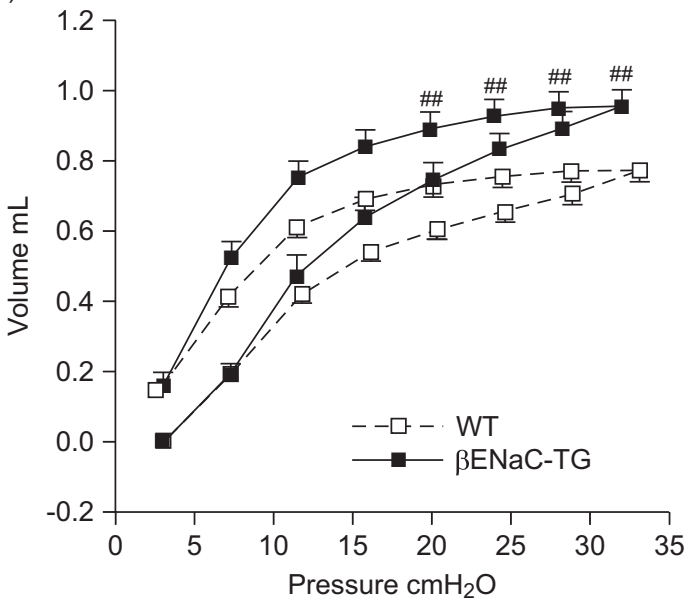

FIGURE 6. Development of emphysema in $\beta$-epithelial $\mathrm{Na}^{+}$channel (ENaC)-transgenic (TG) mice. a) Summary of longitudinal measurements of the density of lung parenchyma, in Hounsfield units (HU), in neonatal to adult wild-type (WT) and $\beta E N a C-T G$ mice. - : median; box: interquartile range; whiskers: 10th and 90th percentiles. $\mathrm{n}=11-20$ mice per group. ${ }^{*}: \mathrm{p}<0.005$ versus age-matched WT (Bonferroni-corrected Mann-Whitney-U test); ": $\mathrm{p}<0.05$ versus WT on day 42 (Kruskal-Wallis ANOVA with post hoc Dunn's test); ${ }^{+}: p<0.05$ versus $\beta E N a C-T G$ on day 42 (Kruskal-Wallis ANOVA with post hoc Dunn's test); *: $p<0.05$ versus WT on day 3 (Kruskal-Wallis ANOVA with post hoc Dunn's test). b, c) Representative lung histology (haematoxylin and eosin staining) from 2-week-old mice showing enlargement of alveoli in c) $\beta E N a C$-TG compared with b) WT mice. Scale bars $=100 \mu \mathrm{m}$. d) Mean linear intercepts were determined in WT and $\beta E N a C-T G$ mice at the ages of 3 days, 2 weeks and 6 weeks. Data are presented as mean \pm SEM. $n=5-8$ mice per group. **: $p<0.01$ versus $\beta E N a C-T G$ on day 3 (one-way ANOVA with post hoc Bonferroni-corrected unpaired $t$-test); $* * *: p<0.001$ versus WT

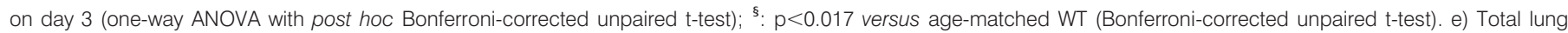
capacity (TLC) and f) pressure-volume curves were determined in 6-week-old $\beta E N a C-T G$ mice and WT littermates. Data are presented as mean \pm SEM. $n=18-20$ per group. ${ }^{f}: \mathrm{p}<0.05$ versus WT (unpaired t-test); ${ }^{\# \#: ~} \mathrm{p}<0.01$ versus corresponding WT (Bonferroni-corrected unpaired t-test).

Our data demonstrate that longitudinal in vivo imaging by VCT has significant advantages over a cross-sectional histopathological approach for phenotyping of lung disease in mice. First, VCT enabled intraindividual monitoring of the development of specific abnormalities in $\beta E N a C-T G$ mice (figs 1, 2 and 4-6). Secondly, longitudinal VCT imaging allowed us to study morphological changes that occurred in deceased $\beta E N a C-T G$ mice prior to death and thus capture valuable information that cannot be obtained by histopathological evaluation. Moreover, VCT provides images of the lung as a whole, whereas histology is typically limited to a small number of lung sections. Finally, by exploiting these advantages, VCT has the potential to reduce the number of experimental mice required for future studies with morphological outcomes. 
In vivo imaging of the lung of small laboratory animals by VCT has several advantages when compared with other imaging techniques. In previous studies, $\mu \mathrm{CT}$ was used to provide highresolution images with possible voxel sizes $<50 \mu \mathrm{m}[18,19,33]$ to study murine models of interstitial lung disease and emphysema [16, 17]. However, $\mu \mathrm{CT}$ requires long scanning times of $\leqslant 30 \mathrm{~min}$ [17], accompanied by high radiation exposure of up to several Gray units [33] and invasive anaesthesia. Thus, there is agreement that the usefulness of $\mu \mathrm{CT}$ for longitudinal in vivo studies is limited [19, 33]. Other investigators employed magnetic resonance imaging (MRI) for visualisation of the pleura and gross pulmonary fluid in rat models of pulmonary inflammation [34, 35]. However, the limited spatial resolution of MRI precludes its use for structural analysis of airways and lung parenchyma in small laboratory animals.

Because the lung has a high inherent tissue contrast, we applied higher photon energies $(80 \mathrm{kV})$ and reduced scan times to several seconds. Using these settings, we demonstrated that the resolution achieved by VCT was sufficient to identify alterations of the lung texture and the larger airways in 3-day-old neonatal mice with a body weight of $\sim 2-3 \mathrm{~g}$. The maximum cumulative dose of 10 subsequent VCT scans was $\sim 230 \mathrm{mGy}$ [23], which was well tolerated by mice in our study and previous studies $[36,37]$, thus allowing noninvasive longitudinal monitoring of lung disease in mice from neonatal to adult ages.

Our study also identified some limitations of VCT in pulmonary imaging of mice. Because the diameter of the small airways [38] is below the spatial resolution of VCT [22], the technique was insensitive in detecting abnormalities in this airway region. Moreover, cardiac and respiratory motion of freely breathing mice probably decreased the signal-to-noise ratio. However, we decided against the use of respiratory or cardiac gating in our experiments [22] for the following reasons. First, most lung alterations of interest in this study affected the organ as a whole, whereas gating for VCT was described mainly to increase the delineation of structures close to the diaphragm [22]. Therefore, the additional scan time, X-ray exposure and anaesthetics required for retrospective gating may have adverse effects on animal survival and lung structure, without providing relevant additional information. Secondly, simultaneous scanning of several animals allowed studies of larger groups, but made gating of respiratory and cardiac function impossible.

In summary, we demonstrate that VCT imaging is suitable for detecting key histopathological features, such as airway mucus obstruction and emphysema, in $\beta \mathrm{ENaC}$-TG mice. Furthermore, VCT studies identified additional abnormalities, which were not previously appreciated by histopathological evaluation, but represent common morphological changes in lung disease in patients with CF including pulmonary infiltrates, atelectasis and air trapping. We conclude that VCT is a sensitive technique for longitudinal noninvasive monitoring of small animal models of $\mathrm{CF}$, and potentially other lung diseases associated with changes in parenchymal density, atelectasis, infiltration or emphysema, such as pneumonia, bronchopulmonary dysplasia and COPD. In these disease models, we predict that VCT will provide a valuable tool for studies of in vivo pathogenesis and may serve as an end-point for preclinical evaluation of novel therapeutic strategies.

\section{SUPPORT STATEMENT}

This study was supported by the Deutsche Forschungsgemeinschaft (grant numbers DFG MA 2081/3-2 and MA 2081/4-1) and the European Commission (grant number MEXT-CT-2004-013666).

\section{STATEMENT OF INTEREST}

A statement of interest for M.A. Mall can be found at www.erj. ersjournals.com/site/misc/statements.xhtml

\section{ACKNOWLEDGEMENTS}

We thank J. Schatterny (University of Heidelberg, Heidelberg, Germany) for expert technical assistance in genotyping of experimental animals.

\section{REFERENCES}

1 Kerem B, Rommens JM, Buchanan JA, et al. Identification of the cystic fibrosis gene: genetic analysis. Science 1989; 245: 1073-1080.

2 Welsh MJ, Ramsey BW, Accurso F, et al. Cystic Fibrosis. 8th Edn. New York, McGraw Hill, 2001.

3 Zuelzer WW, Newton WA Jr. The pathogenesis of fibrocystic disease of the pancreas; a study of 36 cases with special reference to the pulmonary lesions. Pediatrics 1949; 4: 53-69.

4 Gibson RL, Burns JL, Ramsey BW. Pathophysiology and management of pulmonary infections in cystic fibrosis. Am J Respir Crit Care Med 2003; 168: 918-951.

5 Canessa CM, Schild L, Buell G, et al. Amiloride-sensitive epithelial $\mathrm{Na}^{+}$channel is made of three homologous subunits. Nature 1994; 367: 463-467.

6 Stutts MJ, Canessa CM, Olsen JC, et al. CFTR as a cAMP-dependent regulator of sodium channels. Science 1995; 269: 847-850.

7 Mall M, Hipper A, Greger R, et al. Wild type but not deltaF508 CFTR inhibits $\mathrm{Na}^{+}$conductance when coexpressed in Xenopus oocytes. FEBS Lett 1996; 381: 47-52.

8 Mall M, Bleich M, Greger R, et al. The amiloride-inhibitable $\mathrm{Na}^{+}$ conductance is reduced by the cystic fibrosis transmembrane conductance regulator in normal but not in cystic fibrosis airways. J Clin Invest 1998; 102: 15-21.

9 Matsui H, Grubb BR, Tarran R, et al. Evidence for periciliary liquid layer depletion, not abnormal ion composition, in the pathogenesis of cystic fibrosis airways disease. Cell 1998; 95: 1005-1015.

10 Knowles MR, Boucher RC. Mucus clearance as a primary innate defense mechanism for mammalian airways. J Clin Invest 2002; 109: 571-577.

11 Mall MA. Role of cilia, mucus, and airway surface liquid in mucociliary dysfunction: lessons from mouse models. J Aerosol Med Pulm Drug Deliv 2008; 21: 13-24.

12 Mall M, Grubb BR, Harkema JR, et al. Increased airway epithelial $\mathrm{Na}^{+}$absorption produces cystic fibrosis-like lung disease in mice. Nat Med 2004; 10: 487-493.

13 Mall MA, Harkema JR, Trojanek JB, et al. Development of chronic bronchitis and emphysema in $\beta$-epithelial $\mathrm{Na}^{+}$channel-overexpressing mice. Am J Respir Crit Care Med 2008; 177: 730-742.

14 Mall MA. Role of the amiloride-sensitive epithelial $\mathrm{Na}^{+}$channel in the pathogenesis and as a therapeutic target for cystic fibrosis lung disease. Exp Physiol 2009; 94: 171-174.

15 Zhou Z, Treis D, Schubert SC, et al. Preventive but not late amiloride therapy reduces morbidity and mortality of lung disease in $\beta E N a C$-overexpressing mice. Am J Respir Crit Care Med 2008; 178: 1245-1256.

16 Postnov AA, Meurrens K, Weiler $\mathrm{H}$, et al. In vivo assessment of emphysema in mice by high resolution X-ray microtomography. J Microsc 2005; 220: 70-75.

17 Lee HJ, Goo JM, Kim NR, et al. Semiquantitative measurement of murine bleomycin-induced lung fibrosis in in vivo and post mortem conditions using microcomputed tomography: correlation with pathologic scores - initial results. Invest Radiol 2008; 43: 453-460. 
18 Ritman EL. Micro-computed tomography-current status and developments. Annu Rev Biomed Eng 2004; 6: 185-208.

19 Ritman EL. Micro-computed tomography of the lungs and pulmonary-vascular system. Proc Am Thorac Soc 2005; 2: 477-480.

20 Kiessling F, Greschus S, Lichy MP, et al. Volumetric computed tomography (VCT): a new technology for noninvasive, highresolution monitoring of tumor angiogenesis. Nat Med 2004; 10: 1133-1138.

21 Greschus S, Kiessling F, Lichy MP, et al. Potential applications of flat-panel volumetric CT in morphologic and functional small animal imaging. Neoplasia 2005; 7: 730-740.

22 Bartling SH, Stiller W, Grasruck M, et al. Retrospective motion gating in small animal CT of mice and rats. Invest Radiol 2007; 42: 704-714.

23 Gupta R, Grasruck M, Suess C, et al. Ultra-high resolution flatpanel volume CT: fundamental principles, design architecture, and system characterization. Eur Radiol 2006; 16: 1191-1205.

24 Harkema JR, Plopper CG, Hyde DM, et al. Regional differences in quantities of histochemically detectable mucosubstances in nasal, paranasal, and nasopharyngeal epithelium of the bonnet monkey. J Histochem Cytochem 1987; 35: 279-286.

25 Dunnill MS. Quantitative methods in the study of pulmonary pathology. Thorax 1962; 17: 320-328.

26 Vanoirbeek JA, Rinaldi M, De Vooght V, et al. Noninvasive and invasive pulmonary function in mouse models of obstructive and restrictive respiratory diseases. Am J Respir Cell Mol Biol 2009; 42: 96-104.

27 Marcos V, Zhou Z, Yildirim AO, et al. CXCR2 mediates NADPH oxidase-independent neutrophil extracellular trap formation in cystic fibrosis airway inflammation. Nat Med 2010; 16; 1018-1023.

28 Cardoso WV. Lung morphogenesis, role of growth factors and transcription factors. In: Harding R, Pinkerton KE, Plopper CG, eds. The Lung: Development, Aging and the Environment. London, Elsevier, 2004; pp. 3-11.
29 Heussel CP, Herth FJ, Kappes J, et al. Fully automatic quantitative assessment of emphysema in computed tomography: comparison with pulmonary function testing and normal values. Eur Radiol 2009; 19: 2391-2402.

30 Cobos-Correa A, Trojanek JB, Diemer S, et al. Membrane-bound FRET probe visualizes MMP12 activity in pulmonary inflammation. Nat Chem Biol 2009; 5: 628-630.

31 Schubert SC, Trojanek JB, Diemer S, et al. Airways surface liquid depletion causes MMP-12 dependent emphysema in $\beta E N a C$ overexpressing mice. J Cyst Fibros 2009; 8: 53.

32 Gaggar A, Hector A, Bratcher PE, et al. The role of matrix metalloproteases in cystic fibrosis lung disease. Eur Respir J 2011; 38: 721-727.

33 Ford NL, Thornton MM, Holdsworth DW. Fundamental image quality limits for microcomputed tomography in small animals. Med Phys 2003; 30: 2869-2877.

34 Quintana HK, Cannet C, Schaeublin E, et al. Identification with MRI of the pleura as a major site of the acute inflammatory effects induced by ovalbumin and endotoxin challenge in the airways of the rat. Am J Physiol Lung Cell Mol Physiol 2006; 291: L651-L657.

35 Quintana HK, Cannet C, Zurbruegg S, et al. Proton MRI as a noninvasive tool to assess elastase-induced lung damage in spontaneously breathing rats. Magn Reson Med 2006; 56: 1242-1250.

36 Bartling SH, Stiller W, Semmler W, et al. Small animal computed tomography imaging. Curr Med Imaging Rev 2007; 3: 45-59.

37 Drangova M, Ford NL, Detombe SA, et al. Fast retrospectively gated quantitative four-dimensional (4D) cardiac micro computed tomography imaging of free-breathing mice. Invest Radiol 2007; 42: 85-94.

38 Evans CM, Williams OW, Tuvim MJ, et al. Mucin is produced by Clara cells in the proximal airways of antigen-challenged mice. Am J Respir Cell Mol Biol 2004; 31: 382-394. 